\title{
Implementing a nurse-enabled, integrated, shared-care model involving specialists and general practitioners in breast cancer post- treatment follow-up: a study protocol for a phase II randomised controlled trial (the EMINENT trial)
}

Raymond Javan Chan ${ }^{1,2^{*}}$ (D) Jon Emery ${ }^{3}$, Katharine Cuff', Laisa Teleni ${ }^{2}$, Camilla Simonsen ${ }^{1}$, Jane Turner ${ }^{4}$, Monika Janda ${ }^{5}$, Daniel Mckavanagh ${ }^{1}$, Lee Jones ${ }^{6}$, Emma McKinnell', Melissa Gosper ${ }^{7}$, Juanita Ryan ${ }^{8}$, Ria Joseph$^{2}$, Bethany Crowe ${ }^{1}$, Jennifer Harvey ${ }^{9}$, Marissa Ryan ${ }^{10}$, Christine Carrington ${ }^{10}$, Rebecca Nund ${ }^{11}$, Megan Crichton ${ }^{2}$ and Steven McPhail ${ }^{12,13}$

\begin{abstract}
Background: Due to advances in early detection and cancer treatment, 5-year relative survival rates for early breast cancer surpass $90 \%$ in developed nations. There is increasing focus on promotion of wellness in survivorship and active approaches to reducing morbidity related to treatment; however, current models of follow-up care are heavily reliant on hospital-based specialist-led care. This study aims to test the feasibility of the EMINENT intervention for implementing an integrated, shared-care model involving both cancer centre specialists and community-based general practitioners for early breast cancer post-treatment follow-up.
\end{abstract}

Methods: We describe a protocol for a phase II, randomised controlled trial with two parallel arms and 1:1 allocation. A total of 60 patients with early-stage breast cancer will be randomised to usual, specialist-led, follow-up care (as determined by the treating surgeons, medical oncologists, and radiation oncologists) or shared follow-up care intervention (i.e. EMINENT). EMINENT is a nurse-enabled, pre-specified shared-care pathway with follow-up responsibilities divided between cancer centre specialists (i.e. surgeons and oncologists) and general practitioners. The primary outcome is health-related quality of life as measured by the Functional Assessment of Cancer Therapy-Breast Cancer. Secondary outcomes include patient experience, acceptance, and satisfaction of care; dietary, physical activity, and sedentary behaviours; financial toxicity; adherence; health resource utilisation; and adverse events.

(Continued on next page)

\footnotetext{
* Correspondence: raymond.chan@qut.edu.au

'Division of Cancer Services, Proncess Alexandra Hospital, Metro South Health, Brisbane, Queensland, Australia

${ }^{2}$ School of Nursing, Queensland University of Technology, Brisbane, Queensland, Australia

Full list of author information is available at the end of the article
}

C C The Author(s). 2020 Open Access This article is licensed under a Creative Commons Attribution 4.0 International License, which permits use, sharing, adaptation, distribution and reproduction in any medium or format, as long as you give appropriate credit to the original author(s) and the source, provide a link to the Creative Commons licence, and indicate if changes were made. The images or other third party material in this article are included in the article's Creative Commons licence, unless indicated otherwise in a credit line to the material. If material is not included in the article's Creative Commons licence and your intended use is not permitted by statutory regulation or exceeds the permitted use, you will need to obtain permission directly from the copyright holder. To view a copy of this licence, visit http://creativecommons.org/licenses/by/4.0/. The Creative Commons Public Domain Dedication waiver (http://creativecommons.org/publicdomain/zero/1.0/) applies to the data made available in this article, unless otherwise stated in a credit line to the data. 
(Continued from previous page)

Discussion: The trial is designed to identify the barriers to implementing a shared-care model for breast cancer survivors following treatment. Results of this study will inform a definitive trial testing the effects of shared-care model on health-related quality of life of breast cancer survivors, as well as its ability to alleviate the growing demands on the healthcare system.

Trial registration: Australia and New Zealand Clinical Trials Registry ACTRN12619001594112. Registered on 19 November 2019

Keywords: Early breast cancer, Oncology, Shared-care, Quality of life, Protocol, Randomised controlled trial

\section{Administrative information}

The order of the items has been modified to group similar items (see http://www.equator-network.org/reporting-guidelines/spirit-2013-statement-defining-standardprotocol-items-for-clinical-trials/).

\begin{tabular}{|c|c|}
\hline Title $\{1\}$ & $\begin{array}{l}\text { Implementing a nurse-enabled, inte- } \\
\text { grated, shared-care model involving } \\
\text { specialists and general practitioners in } \\
\text { breast cancer post-treatment follow-up: } \\
\text { a study protocol for a Phase II rando- } \\
\text { mised controlled trial (The EMINENT } \\
\text { Trial) }\end{array}$ \\
\hline Trial registration $\{2 \mathrm{a}$ and $2 \mathrm{~b}\}$. & $\begin{array}{l}\text { Registry Name: Australia and New } \\
\text { Zealand Clinical Trials Registry. Trial } \\
\text { Identifier: ACTRN12619001594112 }\end{array}$ \\
\hline Protocol version $\{3\}$ & 24/03/2020, Version 2.0 \\
\hline Funding $\{4\}$ & $\begin{array}{l}\text { Metro South Health SERTA (Study, } \\
\text { Education and Research Trust Account) } \\
\text { grant. }\end{array}$ \\
\hline Author details $\{5 a\}$ & $\begin{array}{l}\text { Raymond Javan Chan, Division of } \\
\text { Cancer Services, Princess Alexandra } \\
\text { Hospital, Metro South Health \& School } \\
\text { of Nursing, Queensland University of } \\
\text { Technology, Brisbane, Queensland, } \\
\text { Australia. Email: raymond.chan@qut.edu. } \\
\text { au } \\
\text { Jon Emery, Department of General } \\
\text { Practice and Centre for Cancer } \\
\text { Research, University of Melbourne, } \\
\text { Melbourne, Victoria, Australia. Email: jon. } \\
\text { emery@unimelb.edu.au } \\
\text { Katharine Cuff, Head of Haematology, } \\
\text { Division of Cancer Services, Princess } \\
\text { Alexandra Hospital, Metro South Health; } \\
\text { Senior lecturer, University of } \\
\text { Queensland; and Clinical Assoc Prof, } \\
\text { Queensland University of Technology, } \\
\text { Brisbane, Queensland, Australia. Email: } \\
\text { Katharine.Cuff@health.qld.gov.au } \\
\text { Laisa Teleni, Research Coordinator, } \\
\text { School of Nursing, Queensland } \\
\text { University of Technology, Brisbane, } \\
\text { Queensland, Australia. Email: laisa. } \\
\text { teleni@qut.edu.au } \\
\text { Camilla Simonsen, Division of Cancer } \\
\text { Services, Princess Alexandra Hospital, } \\
\text { Metro South Health, Brisbane, } \\
\text { Queensland, Australia. Email: Camilla. } \\
\text { Simonsen@health.qld.gov.au } \\
\text { Jane Turner, Faculty of Medicine, } \\
\text { University of Queensland \& Royal } \\
\text { Brisbane and Women's Hospital, } \\
\text { Mund }\end{array}$ \\
\hline
\end{tabular}

\section{Administrative information (Continued)}

Brisbane, Queensland, Australia. Email: jane.turner@uq.edu.au Monika Janda, Centre for Health Services Research, Faculty of Medicine, University of Queensland, Brisbane, Queensland, Australia. Email: m. janda@qut.edu.au

Daniel Mckavanagh, Division of Cancer Services, Princess Alexandra Hospital, Metro South Health, Brisbane,

Queensland, Australia. Email: Daniel. Mckavanagh@health.qld.gov.au Lee Jones, Institute of Health and Biomedical Innovation, Queensland University of Technology, Brisbane, Queensland, Australia. Email: lee. jones@qut.edu.au

Emma McKinnell, Division of Cancer Services, Princess Alexandra Hospital, Metro South Health, Brisbane, Queensland, Australia. Email: Emma. McKinnell@health.qld.gov.au Melissa Gosper, McGrath Foundation \& Princess Alexandra Hospital, Metro South Health, Brisbane, Queensland, Australia. Email: Melissa.Gosper@health. qld.gov.au

Juanita Ryan, Division of Surgery,

Princess Alexandra Hospital, Metro South Health, Brisbane, Queensland, Australia. Email: Juanita.Ryan@health. qld.gov.au

Ria Joseph, School of Nursing, Queensland University of Technology, Brisbane, Queensland, Australia. Email: ria.joseph@qut.edu.au

Bethany Crowe, Division of Cancer Services, Princess Alexandra Hospital, Metro South Health, Brisbane,

Queensland, Australia. Email: bethany. crowe@health.qld.gov.au Jennifer Harvey, Radiation Oncology Centre, Princess Alexandra Hospital, Metro South Health and University of Queensland, Brisbane, Queensland, Australia. Email: Jennifer.Harvey@health. qld.gov.au

Marissa Ryan, Pharmacy Department, Princess Alexandra Hospital, Metro South Health, Brisbane, Queensland, Australia. Email: Marissa.Ryan@health. qld.gov.au

Christine Carrington, Department of Pharmacy, Princess Alexandra Hospital, Metro South Health \& School of 


\section{Administrative information (Continued)}

Pharmacy, University of Queensland, Brisbane, Queensland, Australia. Email: Christine.Carrington@health.qld.gov.au Rebecca Nund, School of Health and Rehabilitation Sciences, University of Queensland, Brisbane, Queensland, Australia.Email: r.nund@uq.edu.au Megan Crichton, School of Nursing, Queensland University of Technology, Brisbane, Queensland, Australia. Email: megan.crichton@qut.edu.au

Steven McPhail, Australian Centre For Health Services Innovation (AusHSI),

Queensland University of Technology, Brisbane, Queensland, Australia \& Clinical Informatics Division, Metro South Health, Brisbane, Queensland, Australia. Email: steven.mcphail@qut. edu.au

Name and contact information for the trial sponsor $\{5 b\}$

Role of sponsor $\{5 c\}$

Professor Raymond Chan

Division of Cancer Services, Princess Alexandra Hospital, Metro South Health Brisbane, Queensland, Australia. And

School of Nursing, Queensland University of Technology, Brisbane, Queensland, Australia.

Email: Raymond.chan@qut.edu.au

The sponsor has absolute authority over securing funding, study design, data analysis, interpretation of data, writing of the report, decision to submit the report for publication.

\section{Introduction}

\section{Background and rationale $\{6 a\}$}

In Australia, breast cancer is the most common cancer in females with an estimated 19,535 new cases annually [1]. With advances in early detection and cancer treatment, such as surgery, post-operative radiotherapy, and pre- or post-operative systemic therapies including cytotoxic chemo-, endocrine, and anti-HER2 antibody therapies, the 5-year relative survival rate for breast cancer is estimated at 91\% [2, 3]. Consequently, in 2018, there were at least 200,000 breast cancer survivors living in Australia [4]. Despite good survival outcomes, breast cancer survivors require supportive care including prevention of cancer recurrence, surveillance for secondary or new primary cancer, and management of a range of long-term bio-psycho-social effects from their cancer diagnosis and treatment. In addition, many cancer survivors need management of comorbidities as they are 2.5 times more likely to develop mental and behavioural problems and almost 1.5 times more likely to develop musculoskeletal conditions, circulatory conditions, and endocrine system disorders compared with non-cancer patients [5]. These health concerns highlight the importance of a comprehensive, well-integrated, patient-

centred model of care for people following completion of breast cancer treatment.

The current models of post-treatment care in Australia are mostly hospital-based and specialist-driven and focus on surveillance for disease recurrence, rather than the holistic care needs of cancer patients. This model of followup care limits the integration between specialist institutions and general practitioners (GPs), overloads the specialist system, and is unsustainable to meet the demands of the ever-growing population of cancer survivors. Specialist-based follow-up carries the burdens of travel and out-of-pocket costs such as those for parking. Those in non-metropolitan areas face more major disruptions to engage in specialist-based follow-up. Therefore, there is a strong case for an integrated, shared post-treatment follow-up care model for breast cancer survivors that involves both cancer specialists as well as care provided in the community by GPs. Such a shared-care model is consistent with Cancer Australia statements [6], the Optimal Care Pathway for Breast Cancer [7], and international guidelines [8]. In addition, the literature suggests that such a model is feasible, acceptable, safe, and more cost effective and patient-centred than current models used within Australia [9, 10].

Despite the promising evidence base, a shared followup care model in which specialists in the acute cancer care setting and GPs collaborate is not routinely implemented across Australia and many developed nations. Barriers to such shared care include the lack of a coordination between multiple providers, lack of patient and provider knowledge about the benefits of shared care and how to implement it, insufficient or delayed communication between cancer specialists and GPs, and lack of awareness of available support such as funding models, tools, and resources [11, 12]. These barriers could be overcome if a Specialist Cancer Nurse advises stakeholders (patient and GPs) of the benefits of sharedcare, facilitates effective and timely care coordination, and acts as the conduit between the specialist cancer multidisciplinary team and the GPs at key transition time points, such as completion of definitive primary and adjuvant treatment [13].

\section{Objectives $\{7\}$}

The objective of the study is to test the feasibility of a prospective, pragmatic randomised controlled trial (RCT) of the EMINENT intervention-a nurse-enabled, integrated, shared-care model involving cancer specialists and GPs for early breast cancer post-treatment follow-up.

\section{Trial design $\{8\}$}

This phase II pilot RCT aims to assess the feasibility of a larger definitive clinical trial. Outcome data will be 
collected at four timepoints (or five if Booster Nurse Clinic is attended): $\left(t_{1}\right)$ baseline (at enrolment \pm prior to Booster Nurse Clinic, if relevant), $\left(t_{2}\right) 3$ months, $\left(t_{3}\right) 6$ months, and $\left(t_{4}\right) 12$ months.

\section{Methods: participants, interventions, and outcomes \\ Study setting $\{9\}$}

This study is conducted in a large, Australian metropolitan tertiary teaching hospital and general practices.

\section{Eligibility criteria $\{10\}$}

The study population consists of patients with earlystage breast cancer (i.e. no-distant metastases) or ductal carcinoma in situ (DCIS). Patients will be eligible to participate from 2 weeks prior to completion of definitive treatment (surgery or adjuvant chemotherapy) and up to 18 months after completion of treatment. Patients meeting all of the following criteria are eligible for inclusion: diagnosis of curable, early breast cancer; receiving care at the Princess Alexandra Hospital; able to speak and read English; 18 years of age or older; ambulatory at the time of recruitment; Eastern Cooperative Oncology Group (ECOG) performance status 0 or 1; able to nominate a GP or GP clinic to be involved in their follow-up; and access to a telephone. Patients meeting any of the following criteria are excluded: presence of severe mental, cognitive, or physical conditions that would limit the patient's ability to provide informed consent.

\section{Who will take informed consent? $\{26 \mathrm{a}\}$}

Potential participants are identified by the research nurse or treating clinician during multidisciplinary team meetings. Participants are approached by their treating clinicians to gauge their interest in the study and gain verbal consent to being approached by the research team. Participants are then contacted by the research nurse, screened for eligibility, and provided with study information, and after a time of reflection (at least $24 \mathrm{~h}$ ), they sign the consent form with the research nurse. Table 3 outlines the different phases of the study and data collection.

\section{Additional consent provisions for collection and use of participant data and biological specimens $\{26 \mathrm{~b}\}$}

Consent to access Medicare and Pharmaceutical Benefits Scheme (PBS) data on service use that qualifies under the Medicare Benefits Schedule (MBS) will be obtained, including relevant claims details (date of service, Medicare item number, and description) and costs details.

\section{Interventions}

Explanation for the choice of comparators $\{6 \mathrm{~b}\}$

Survivorship care of breast cancer survivors following completion of treatment is an important issue, especially in light of improving survival rates [2]. The shared-care model between specialists and GPs focusses on the complex care needs of breast cancer survivors, rather than solely on disease recurrence, and may influence patient health outcomes and service outcomes [10].

\section{Intervention description $\{11 \mathrm{a}\}$}

\section{Arm 1}

The control group will receive usual follow-up care supplemented with a survivorship booklet on Living Well After Cancer published by Cancer Council Australia [14]. The usual care follow-up arrangement is a specialist-led model as determined by the treating surgeon, medical oncologist, and radiation oncologist. This specialist-led follow-up care is not standardised and with follow-up activities and schedules depending on individual patient needs and the discretion of the treating clinicians.

\section{Arm 2}

EMINENT is a multi-faceted intervention that includes a pre-specified shared-care pathway for post-treatment follow-up. The design of the EMINENT intervention is informed by a number of Cancer Australia statements, the Optimal Care Pathway [15], the self-efficacy model $[16,17]$, the Capabilities for Supporting Prevention and Chronic Condition Self-Management framework [18], and our extensive pilot work including a systematic review [19] and observational studies [20-23]. Table 1 outlines the active ingredients of the EMINENT intervention.

After enrolment, participants who have completed chemotherapy and radiotherapy or those who will receive aromatase inhibitor will participate in a 20 -min telehealth cancer pharmacist consultation for medication reconciliation and education prior to Specialist Nurse consultation.

A 30-60-min consultation with a Specialist Cancer Nurse is then conducted to provide a treatment summary, the shared follow-up care appointment schedule, and survivorship patient education (including the survivorship booklet on Living Well After Cancer published by Cancer Council Australia [14]) and to codevelop a draft Survivorship Care Plan (SCP). The SCP includes up to three SMART (Specific, Measurable, Achievable, Realistic, and Timely) goals that are developed by the nurse and patient in partnership using motivational interviewing and self-efficacy techniques. Due to the recent COVID-19 pandemic, where there are delays of 3-18 months before GP involvement, a second 
Table 1 Active ingredients of the EMINENT model of care intervention for patients allocated to receive the EMINENT intervention

\begin{tabular}{|c|c|c|}
\hline Active ingredient & Personnel involved & Specific activities \\
\hline $\begin{array}{l}\text { Nurse Clinic ( } 30-60 \text { min) } \\
\text { Mode: face to face or } \\
\text { telehealth }\end{array}$ & Specialist Cancer Nurse $\mathrm{a}^{\mathrm{a}}$ and patient & $\begin{array}{l}\text { - Treatment summary } \\
\text { - Survivorship care planning } \\
\text { - Collaborative planning for health goals } \\
\text { - Post-treatment education }\end{array}$ \\
\hline $\begin{array}{l}\text { Booster Nurse Clinic }{ }^{\mathbf{b}} \\
\text { (10-30 min) } \\
\text { Mode: face to face } \\
\text { or telehealth }\end{array}$ & Specialist Cancer Nurse $\mathrm{a}^{\mathrm{a}}$ and patient & $\begin{array}{l}\text { - Survivorship care planning } \\
\text { - Collaborative planning for health goals }\end{array}$ \\
\hline $\begin{array}{l}\text { Pharmacist Consultation } \\
\text { (20 min) } \\
\text { Mode: face to face or telehealth }\end{array}$ & Cancer Pharmacist and patient & $\begin{array}{l}\text { - Medication reconciliation } \\
\text { - Medication education }\end{array}$ \\
\hline $\begin{array}{l}\text { Case conference with GP } \\
\text { (5-30 min) } \\
\text { Mode: teleconference }\end{array}$ & $\begin{array}{l}\text { Specialist Cancer Nurse }{ }^{a} \text { and GP } \\
( \pm \text { one more healthcare team member) }\end{array}$ & $\begin{array}{l}\text { - Nurse presents treatment summary and survivorship care plan } \\
\text { - Follow-up responsibilities of the GP negotiated } \\
\text { - GP questions answered } \\
\text { - Additional education and support provided to other healthcare } \\
\text { team members (e.g. Practice Nurse) }\end{array}$ \\
\hline $\begin{array}{l}\text { Shared follow-up care } \\
\text { Mode: face to face or } \\
\text { telehealth }\end{array}$ & $\begin{array}{l}\text { Cancer specialist, GP, and Specialist } \\
\text { Cancer Nurse }{ }^{a} \text { and patient }\end{array}$ & $\begin{array}{l}\text { - Cancer specialist reviews patient, orders mammogram, and completes } \\
\text { full physical examination every } 6 \text { months for } 2 \text { years post-diagnosis, } \\
\text { then every } 12 \text { months up to } 5 \text { years post-diagnosis } \\
\text { - GP reviews patient as per survivorship care plan at least every } 12 \text { months } \\
\text { (e.g. for general health and comorbidity management, chronic disease } \\
\text { management planning, psychosocial screening, management of cancer } \\
\text { treatment toxicities and cancer-related symptoms, allied health referrals) } \\
\text { - GP contacts nurse interventionist with any patient concerns }\end{array}$ \\
\hline
\end{tabular}

${ }^{a}$ Medical Oncology Clinical Nurse Consultant, Breast Care Nurse, or McGrath Breast Care Nurse

${ }^{b}$ Offered to patients who have delays in GP involvement greater than 3 months (up to 18 months)

'Offered to patients who have completed chemotherapy and/or are scheduled to receive aromatase inhibitor, or have completed surgery and radiotherapy

'booster' Specialist Cancer Nurse consultation is offered to patients to update the SCP. The treatment summary and draft SCP is provided to the GP.

Within 4 weeks of the Specialist Cancer Nurse consultation, a 5-30-min case conference between the Specialist Cancer Nurse and the patient's nominated GP is completed to communicate the treatment summary and shared follow-up care schedule and to finalise the SCP and negotiate the GP's role in facilitating the SCP goals. The GP may propose changes or express if they are not willing to take part in specific care activities outlined in the SCP. The finalised SCP is then filed in the patient's medical records and provided to the patient and the GP.

The shared, follow-up care schedule consists of 6monthly patient appointments with a cancer centre specialist and annual appointments with the GP for 2 years post-diagnosis. Following this, the schedule consists of alternating 6 monthly appointments with a cancer centre specialist and GP for up to 5 years post-diagnosis. At 5 years post-diagnosis, patients are discharged to the care of the GP, as per usual care. The GP appointments will focus on reviewing the SCP; promoting general health; primary prevention, screening, and management of comorbidities; psychosocial health; cancer treatment toxicities; cancer-related symptoms; chronic disease management planning; and allied health referrals. The GP has direct telephone access to the Specialist Cancer
Nurse in case of concerns or escalation for acute care review. The cancer centre specialist appointments focus on surveillance activities such as physical examination and imaging (i.e. annual mammogram).

\section{Criteria for discontinuing or modifying allocated interventions $\{11 \mathrm{~b}\}$}

The presence of any of the following criteria constitutes cause for the withdrawal of the participant: altered mental capacity resulting in inability to provide continuing informed consent, notification from treating oncologist and/or GP that the participant is not deemed to have the capacity to consent, and recurrence or progressive disease or death.

\section{Strategies to improve adherence to interventions $\{11 \mathrm{c}\}$}

Fidelity of the intervention will be assessed using the framework for behavioural interventions recommended by the National Institute of Health $(\mathrm{NIH})[24,25]$ as outlined in Table 2.

\section{Relevant concomitant care permitted or prohibited during the trial $\{11 \mathrm{~d}\}$}

No concomitant care or intervention is prohibited during the trial. 


\section{Provisions for post-trial care $\{30\}$}

There is no ancillary or post-trial care for participants in this trial. However, it is expected that the SCP generated will have the value of informing longer term updates of the SCP and future survivorship care.

\section{Outcomes $\{12\}$}

The feasibility outcomes are recruitment and acceptability of the intervention. The primary endpoint is healthrelated quality of life (HRQoL) as measured by Functional Assessment of Cancer Therapy-Breast Cancer (FACT-B) [26] at baseline, 3, 6, and 12 months post-enrollment. The 37 -item FACT-B is a valid and reliable tool for use in cancer survivors undergoing as well as beyond treatment and has been demonstrated to be sensitive to changes over time [27]. A total score as well as scores for each of the five subscales (physical, social/family, emotional, functional wellbeing, additional breast cancer concerns) are calculated, where higher scores indicate higher quality of life. FACT-B captures key domains of HRQoL and key symptoms that are relevant to the study population and sensitive to the EMINENT intervention.

Additional outcomes include a range of patientreported secondary endpoints, and process outcomes related to implementation as shown in Table 3. Participants of the intervention group, as well as their nominated carer, breast cancer nurses, GPs, and other healthcare providers including other nurses, and hospital- and community-based rehabilitation providers will be invited to participate in a semi-structured interview. Open ended questions (Online Supplementary Material 1) will explore key factors that facilitate or hinder the implementation of the EMINENT intervention.

\section{Participant timeline $\{13\}$}

\section{Sample size $\{14\}$}

In this pilot study, we will recruit 30 patients per arm in order to provide initial insights into the intervention feasibility and protocol as well as preliminary effect size estimates. The aim of this study is not hypothesis testing, and the power level is therefore not a valid consideration for sample size $[28,29]$. The sample size for this study $(n=60)$ falls within the range of sample size recommendations for pilot studies of this nature $[28,29]$.

\section{Recruitment $\{15\}$}

Participants are recruited through the hospital cancer outpatient clinics and therapy units. Research nurses and designated health professionals identify potential participants. Potential participants are reviewed by a member of the treating team and asked if they would like to be approached by a research nurse or designated

Table 2 Intervention fidelity strategies (adapted)

\begin{tabular}{|c|c|}
\hline Goal & Strategies \\
\hline Training providers & $\begin{array}{l}\text { Specialist Cancer Nurses will be trained to standardise the delivery of the intervention to study participants. } \\
\text { Training includes provision of study manual containing } \\
\text { - Generic study information: standard operating procedures, study overview, reporting and documentation } \\
\text { guidelines, communication flowchart, rationale for the study treatment, completion of survivorship care plan, } \\
\text { self-management goal setting, and health coaching } \\
\text { - Specialist Cancer Nurse-specific information: job description, intervention protocol, quality assurance, and } \\
\text { monitoring } \\
\text { An 8-h training program will be delivered by Experts in Cancer Survivorship and motivational interviewing. The } \\
\text { program includes the National Cancer Nursing Education (EdCAN) learning module on survivorship, related } \\
\text { literature, didactic presentations, and roleplay covering: basic concepts of quality cancer survivorship care, } \\
\text { components of a high-quality treatment summary and survivorship care plan; provision of self-management } \\
\text { support (including collaborative goal setting; motivational interviewing); and MBS item numbers that facilitate } \\
\text { the proposed Model of Care. }\end{array}$ \\
\hline Delivery of intervention & $\begin{array}{l}\text { Intervention procedures are monitored through completion of intervention component checklists to ensure that } \\
\text { the intervention is delivered as intended. Intervention checklists are completed during Clinics and GP Case } \\
\text { Conferences to track protocol deviations across Specialist Cancer Nurses and study Arms. The intervention fidelity } \\
\text { is closely monitored and discussed during the weekly } 30 \text {-min meeting for the first } 3 \text { months of the trial between } \\
\text { the Specialist cancer Nurses, research nurses, and investigators. } \\
\text { Minimising contamination between conditions by training interventionists to address participant questions about } \\
\text { randomisation and their assigned condition using non-biased explanations. }\end{array}$ \\
\hline Receipt of intervention & $\begin{array}{l}\text { The SCP serves as a resource for a participant to understand and refer to whenever they are unsure of follow-up } \\
\text { schedule and collaborative goal setting. }\end{array}$ \\
\hline Enactment of treatment skills & $\begin{array}{l}\text { Enactment of treatment skills includes processes to monitor and improve participant ability to perform treatment- } \\
\text { related behavioural skills and cognitive strategies in relevant real-life settings as intended. This goal will be achieved } \\
\text { by ensuring participants are aware of the follow-up schedules and responsibilities of all health professionals, ensuring } \\
\text { participants will have a copy of the completed SCP including all care responsibilities and goals set for the individual, } \\
\text { and checking in with participants once in the first week into the model, then monthly/bimonthly until the end of the } \\
\text { trial period as resources allow. }\end{array}$ \\
\hline
\end{tabular}


Table 3 Schedule for data collection during the EMINENT trial

\begin{tabular}{|c|c|c|c|c|c|c|c|}
\hline \multirow[b]{3}{*}{ Timepoint } & \multicolumn{7}{|c|}{ Study period } \\
\hline & \multirow{2}{*}{$\begin{array}{l}\text { Enrolment } \\
-t_{1}\end{array}$} & \multirow{2}{*}{$\begin{array}{l}\text { Allocation } \\
0\end{array}$} & \multicolumn{4}{|c|}{ Post-allocation } & \multirow{2}{*}{$\begin{array}{l}\text { Close-out } \\
t_{x}\end{array}$} \\
\hline & & & $t_{1}$ & $t_{2}$ & $t_{3}$ & $t_{4}$ & \\
\hline \multicolumn{8}{|l|}{ Enrolment } \\
\hline Eligibility screen & $x$ & & & & & & \\
\hline Informed consent & $x$ & & & & & & \\
\hline Allocation & & $x$ & & & & & \\
\hline \multicolumn{8}{|l|}{ Interventions } \\
\hline \multicolumn{8}{|l|}{ EMINENT } \\
\hline \multicolumn{8}{|l|}{ Usual Care } \\
\hline \multicolumn{8}{|l|}{ Primary endpoints } \\
\hline Health-related quality of life & & & $\mathrm{X}$ & $\mathrm{X}$ & $\mathrm{x}$ & $\mathrm{x}$ & \\
\hline \multicolumn{8}{|l|}{ Secondary endpoint } \\
\hline Patient experience of care & & & $\mathrm{x}$ & & & & \\
\hline Dietary behaviours & & & $\mathrm{x}$ & $\mathrm{x}$ & $\mathrm{x}$ & $\mathrm{X}$ & \\
\hline Physical activity and sedentary behaviours & & & $\mathrm{X}$ & $\mathrm{X}$ & $\mathrm{X}$ & $\mathrm{X}$ & \\
\hline Financial toxicity & & & $\mathrm{x}$ & & $\mathrm{x}$ & $\mathrm{x}$ & \\
\hline Adherence to clinical assessments & & & & & & & $\mathrm{x}$ \\
\hline Health resource utilisation & & & & $\mathrm{x}$ & $\mathrm{x}$ & $\mathrm{x}$ & $\mathrm{x}$ \\
\hline Satisfaction of care & & & & & & $\mathrm{X}$ & \\
\hline Acceptance of intervention & & & & & & $\mathrm{X}$ & \\
\hline \multicolumn{8}{|l|}{ Participant characteristics } \\
\hline Demographics & & & $\mathrm{x}$ & & & & \\
\hline Clinical characteristics & & & $\mathrm{x}$ & & & & \\
\hline \multicolumn{8}{|l|}{ Process outcomes } \\
\hline Completion of intervention component & & & & & & $\mathrm{x}$ & \\
\hline Clinical encounters at cancer centre & & & & & & & $\mathrm{x}$ \\
\hline Referrals back to acute care & & & & & & & $\mathrm{x}$ \\
\hline GP and specialist visits & & & & & & & $\mathrm{x}$ \\
\hline
\end{tabular}

$t_{1}$ baseline, $t_{2} 3$ months, $t_{3} 6$ months, $t_{4} 12$ months

health professionals for consent to participate in the study. Participants are given as much time as possible to consider their participation and are encouraged to take the information away and discuss participation in the trial with family, friends, and their GP if they so wish to. Participants are also encouraged to ask the research nurses, their treating doctors, or nursing staff any questions in relation to their participation.

\section{Assignment of interventions: allocation}

\section{Sequence generation $\{16 \mathrm{a}\}$}

Computer-generated random numbers are used to allocate participants in a 1:1 ratio by a researcher not involved in recruitment, intervention implementation, or data collection. Randomisation is blocked using random permuted blocks of eight and four to ensure that the groups are balanced periodically within stratification groups. Stratification groups include patients who have received (1) surgery only, (2) surgery and radiation only, (3) surgery and chemotherapy \pm radiation and are Her2 negative, and (4) surgery and chemotherapy \pm radiation and are Her 2 positive. These stratification groups were chosen, with clinician input, to allow learnings for patients with different treatment pathways with different follow-up needs to inform the future definitive trial.

\section{Concealment mechanism \{16b\}}

Allocation sequence is implemented using sequentially numbered opaque, sealed envelopes. Envelopes are only accessed by the research nurse to randomise the patient once recruitment and baseline data has been collected. 


\section{Implementation $\{16 \mathrm{c}\}$}

Allocation sequence is generated by a researcher not involved in recruitment or data collection. Patients are enrolled by a research nurse who collects baseline data prior to randomisation. Enrolling nurses assign participants to the intervention after baseline data collection.

\section{Assignment of interventions: blinding Who will be blinded $\{17 \mathrm{a}\}$}

After assignment to the intervention, only outcome assessors are blinded to group allocation. Where participants opt to complete their data collection by phone, they are advised not to reveal their group allocation to the outcome assessor. Due to the nature of the intervention, no participants or treating clinicians are blinded.

\section{Procedure for unblinding if needed $\{17 b\}$}

No unblinding procedures required as only outcome assessors and data analysts are blinded.

\section{Data collection and management}

\section{Plans for assessment and collection of outcomes $\{18 \mathrm{a}\}$}

Patient-reported outcomes are self-administered using online surveys or administered in person or via telephone with an outcome assessor trained in the administration of the study instruments. The description of study instruments is listed in Table 3.

The primary outcome is HRQoL as measured by Functional Assessment of Cancer Therapy-Breast Cancer (FACT-B) [26]. This validated and reliable instrument is well-used in cancer survivors undergoing and beyond treatment [27], and it captures key domains of HRQoL and key symptoms that are relevant to the EMINENT intervention.

The secondary outcomes are listed below:

- Patient experience of care as measured by the Picker Patient Experience 15 (PPE-15) questionnaire [30]. The PPE-15 highlights aspects of care that need improvement to monitor performance and care. It consists of 15 questions distributed to seven dimensions of care: respect, coordination, information/communication/education, physical comfort, emotional support, involvement of relatives, and transitions to community [31].

- Dietary behaviours, specifically usual vegetable intake and usual fruit intake, as measured by two short dietary questions from the National Nutrition Survey [32], which have been validated in the Australian population. Both questions discriminate between groups with significantly different fruit and vegetable intakes. In administering these questions, information about which foods are included as vegetables and fruits is provided and serve sizes are described.

- Physical activity as measured by the Active Australia Survey [33] which is designed to measure participation in leisure-time physical activity, and a single item from the International Physical Activity Questionnaire [34] will be used to measure sedentary behaviours.

- Financial toxicity as measured by the 11 -item Comprehensive Score for financial Toxicity (COST)-Functional Assessment of Chronic Illness Therapy (FACIT) tool [35]. This tool is valid and reliable in measuring financial toxicity in patients with cancer [36].

- Adherence to clinical assessments including annual mammography, annual physical examination, and endocrine therapy as measured by hospital records.

- Emergency presentations and hospitalizations as recorded from hospital records.

- Satisfaction of care as measured by a 0-10 numerical analogue scale with 0 being the least satisfied and 10 being the most satisfied, supplemented with short, structured qualitative questions.

- Process outcomes, including completion of intervention components, as measured by completion of intervention materials such as SCPs and checklists, number and length of clinical encounters recorded from MBS data and hospital records, and barriers and facilitators to implementation as explored through semi-structured interviews with patient participants, their nominated carer, breast cancer nurses, GPs, and other healthcare providers including other nurses, and hospital- and community-based rehabilitation providers.

- Health resource utilisation assessing both health service use and participant out-of-pocket costs including MBS and PBS administrative data sets. These data inform participants' utilisation of services that qualify under the MBS as well as medications dispensed under the PBS. It is planned that the economic evaluation may be reported separately from the main trial.

\section{Plans to promote participant retention and complete follow-up \{18b\}}

Participants who deviate from the protocol are not withdrawn from the trial. Participants who withdraw from the trial nominate the degree to which they withdraw (i.e. whether they withdraw from active data collection \pm passive data collection such as MBS/PBS data). 


\section{Data management $\{19\}$}

All participant characteristic and outcome data are entered directly into REDCap (Research Electronic Data CAPture - Vanderbilt University, hosted at Queensland University of Technology) by the research nurse \pm the participants through self-administered online survey. To ensure data quality, the database is designed with branching logic, data validation, and range checks for data values, where possible.

All source data, clinical records, and laboratory data relating to the study will be archived at the clinical site as appropriate for 15 years after the completion of the study. All data will be available for retrospective review or audit. No study document will be destroyed without prior written agreement between the responsible organisation and the investigator. If the investigator wishes to assign the study records to another party or move them to another location, he/she must notify the responsible organisation in writing of the new responsible person and/ or the new location.

\section{Confidentiality $\{27\}$}

Data on potential participants is recorded, including reasons for ineligibility or refusal to participate. Participants are only identified by a unique participant study number on the case report forms and other study documents. Other study-related documents (e.g. signed consent form, participant log) are kept in strict confidence by the investigator.

Participants are informed that data is held on file by the responsible organisations and that these data may be viewed by staff including the study project manager and by external auditors on behalf of the responsible organisations and appropriate regulatory authorities (to include reviewing Human Research Ethics Committee (HREC) and the Research Governance Officers). Participants will be identified in publication and conference presentation reports only in aggregated form. All participant data will be held in strict confidence.

Plans for collection, laboratory evaluation, and storage of biological specimens for genetic or molecular analysis in this trial/future use $\{33\}$

Not applicable. There is no collection of biological specimens in the current trial.

\section{Statistical methods}

Statistical methods for primary and secondary outcomes $\{20 a\}$

Descriptive statistics will be used to report on feasibility and process-related elements (e.g. recruitment, intervention, retention rates) as well as clinical and resource outcomes. Preliminary effect size estimates for patient and resource use outcomes will be calculated following intention-to-treat principles using generalised linear mixed models. The distribution of the mixed models will be chosen as appropriate for the data, for example, a linear model for scale data or a Poisson for count data. Models will be adjusted for variables used in stratification of the randomisation process. Residuals of all models will be examined for statistical assumptions using descriptive statistics and plots.

Interim analyses $\{\mathbf{2 1 b}\}$

Not applicable. No interim analysis is planned.

\section{Methods for additional analyses (e.g. subgroup analyses) \{20b\}}

All qualitative interviews with participants assigned to the EMINENT intervention are audio-recorded and transcribed verbatim for analysis guided by the Consolidated Framework for Implementation Research [24].

Methods in analysis to handle protocol non-adherence and any statistical methods to handle missing data $\{20 \mathrm{c}\}$

Preliminary effect size estimates for patient and resource use outcomes will be calculated following intention-totreat principles using generalised linear mixed models. Patterns of missing data will be examined using chisquare and $t$ tests. Missing data for the outcomes will be accounted for by using mixed models allowing the use of each available case by computing maximum likelihood estimates.

Plans to give access to the full protocol, participant leveldata, and statistical code $\{31 \mathrm{c}\}$

Not applicable. There are no plans for granting public access of the full protocol, participant-level dataset, or statistical code.

\section{Oversight and monitoring}

Composition of the coordinating centre and trial steering committee $\{5 \mathrm{~d}\}$

The Chief investigators are the trial steering committee that will provide all governance to the conduct of the study.

Composition of the data monitoring committee, its role, and reporting structure $\{21 \mathrm{a}\}$

Not applicable. There is no data monitoring committee established for this pilot trial.

\section{Adverse event reporting and harms $\{22\}$}

An adverse event (AE) is any event, side effect, or other untoward medical occurrences that occur in conjunction with the use of the study intervention in humans, whether or not considered to have a causal relationship to the interventions. An $\mathrm{AE}$ can, therefore, be any 
unfavourable and unintended sign (that could include a clinically significant abnormal laboratory finding), symptom, or disease temporally associated with the use of the study intervention, whether or not considered related to the intervention. Conditions recognised as being excluded from $\mathrm{AE}$ reporting are as follows: any event, side effect, or other medical occurrences that are anticipated because of the normal course of treatment (standard care). There are no known side effects/adverse events associated with the proposed model of care intervention [9]. Due to the nature of this intervention, there will be no reporting of AE.

\section{Frequency and plans for auditing trial conduct $\{23\}$}

There are no plans for auditing trial conduct beyond the independent research governance requirements and annual reporting to the HREC.

\section{Plans for communicating important protocol} amendments to relevant parties (e.g. trial participants, ethical committees) $\{25\}$

All agreed protocol amendments are clearly recorded on a protocol amendment form and are signed and dated by the original protocol approving signatories. All protocol amendments will be submitted to the institutional HREC for approval before implementation. The only exception will be when the amendment is necessary to eliminate an immediate hazard to the trial participants. In this case, the necessary action will be taken first, with the relevant protocol amendment following shortly thereafter. Once HREC approval has been granted, investigators and the ANZCTR will be updated.

\section{Dissemination plans $\{31 \mathrm{a}\}$}

It is intended that the findings from this trial will be disseminated at academic and professional conferences and via a manuscript submission to a peer-reviewed journal. Participants will be identified in such reports only in aggregate or by study identification number, gender, and age. There are no publication restrictions.

\section{Discussion}

Despite the strong case for a shared, follow-up care model for breast cancer survivors involving cancer specialists and GPs, barriers to shared care mean that it is not routinely implemented across Australia. These include the need for coordination across multiple providers, the need for improved patient and provider knowledge about the benefits of shared care and how to implement it, insufficient or delayed communication between cancer specialists and GPs, and lack of awareness of available support such as funding models, tools, and resources $[11,12]$. The current study aims to address these barriers using a Specialist Cancer Nurse to advise stakeholders of the benefits of shared care (patient and GPs), facilitate effective and timely care coordination, and act as the conduit between the specialist cancer multidisciplinary team and the GPs.

Practical issues for this trial include estimating the time required to coordinate the trial across multiple providers including engaging GPs and fidelity with the intervention components. The proposed study will provide important information on the feasibility of a definitive phase 3 trial for implementing a nurseenabled, integrated, shared-care model involving cancer specialists and GPs for early breast cancer posttreatment follow-up. The information collected through the trial, qualitative interviews, and economic evaluations are crucial in guiding the development of such a trial.

\section{Trial status}

The protocol published here is version 2.0 dated 24 March 2020. The trial began recruitment on 3 December 2019 and is expected to continue until 20 November 2021.

Trial registration: Australia and New Zealand Clinical Trials Registry, ACTRN12619001594112. Registered on 19 November 2019, https://www.anzctr.org.au/Trial/ Registration/TrialReview.aspx?id=378690\&isReview=true.

\section{Supplementary information}

Supplementary information accompanies this paper at https://doi.org/10. 1186/s13063-020-04740-1.

Additional file 1. Interview Guide for the Semi-Structured Interviews with Patients/Family Members and Health Professionals.

\section{Abbreviations}

HRQoL: Health-related quality of life; GPs: General practitioners; DCIS: Ductal carcinoma in situ; REDCap: Research Electronic Data Capture; ECOG: Eastern Cooperative Oncology Group; PBS: Pharmaceutical Benefits Scheme; MBS: Medicare Benefits Schedule; SCP: Survivorship Care Plan; FACTB: Functional Assessment of Cancer Therapy_-Breast Cancer; PPE-15: Patient Experience of Care; AAS: Active Australia Survey; IPAQ: International Physical Activity Survey; COST-FACIT: Comprehensive Score for Financial ToxicityFunctional Assessment of Chronic Illness Therapy; SACQ: Self-administered Comorbidity Questionnaire; CCl: Charleston Comorbidity Index; HREC: Human Research Ethics Committee

\section{Acknowledgements}

The authors acknowledge the contribution of Drs Kathryn Middleton, Wen Xu, Kate Roberts, Vladimir Andelkovic, Margo Lehman, and Tao Mai; the contribution of Primary Care Collaborative Cancer Clinical Trials Group; and support from service executives including Professor Euan Walpole, Adj Associate Professor Leanne Stone, and Dr. Doreen Tapsall.

\section{Authors' contributions $\{31 \mathrm{~b}\}$}

$\mathrm{RC}$ is the chief investigator; he conceived the study, secured funding, and led the proposal and protocol development. JE, JH, KC, SM, LT, CS, MJ, LJ, CC $M R$, and DK contributed to the study design and to the development of the protocol. JT is responsible for training of intervention nurses. CS, EM, MG, and $G R$ are responsible for delivering the intervention. MR and $C C$ are 
responsible for delivering the pharmacist consult. All authors have provided input and have read and approved the final manuscript.

\section{Funding \{4\}}

This study is funded by 2019 Metro South Health Research Support Scheme Project Grant (funded by the Metro South Study, Education and Research Trust Account (SERTA)). The funding body had no role in the design of the study and will not have a role in the collection, analysis, and interpretation of data or in writing the manuscript.

\section{Availability of data and materials $\{29\}$}

There are no limitations on investigator access to the trial dataset. The datasets generated and/or analysed during the current study are not going to be made publicly available but will be made available from the corresponding author on reasonable request.

\section{Ethics approval and consent to participate $\{24\}$}

This study is approved by the Metro South Hospital and Health Services Human Research Ethics Committee (HREC/2019/QMS/51956). Written informed consent will be obtained from all participants.

\section{Consent for publication $\{32\}$}

Not applicable. No details, images, or videos relating to an individual person will be published, as all data will be presented in aggregate.

\section{Competing interests $\{28\}$}

The authors declare that they have no competing interests.

\section{Author details}

${ }^{1}$ Division of Cancer Services, Proncess Alexandra Hospital, Metro South Health, Brisbane, Queensland, Australia. ${ }^{2}$ School of Nursing, Queensland University of Technology, Brisbane, Queensland, Australia. ${ }^{3}$ Department of General Practice and Centre for Cancer Research, University of Melbourne, Melbourne, Victoria, Australia. ${ }^{4}$ Faculty of Medicine, University of Queensland \& Royal Brisbane and Women's Hospital, Brisbane, Queensland, Australia. ${ }^{5}$ Centre for Health Services Research, Faculty of Medicine, University of Queensland, Brisbane, Queensland, Australia. ${ }^{6}$ Institute of Health and Biomedical Innovation, Queensland University of Technology, Brisbane, Queensland, Australia. ${ }^{7}$ McGrath Foundation \& Princess Alexandra Hospital, Metro South Health, Brisbane, Queensland, Australia. ${ }^{8}$ Division of Surgery, Princess Alexandra Hospital, Metro South Health, Brisbane, Queensland, Australia. ${ }^{9}$ Radiation Oncology Centre, Princess Alexandra Hospital, Metro South Health and University of Queensland, Brisbane, Queensland, Australia ${ }^{10}$ Pharmacy Department, Princess Alexandra Hospital, Metro South Health, Brisbane, Queensland, Australia. ${ }^{11}$ School of Health and Rehabilitation Sciences, University of Queensland, Brisbane, Queensland, Australia.

${ }^{12}$ Australian Centre For Health Services Innovation (AusHSI), Queensland University of Technology, Brisbane, Queensland, Australia. ${ }^{13}$ Clinical Informatics Division, Metro South Health, Brisbane, Queensland, Australia.

\section{Received: 19 May 2020 Accepted: 16 September 2020}

\section{Published online: 15 October 2020}

\section{References}

1. Australian Institute of Health and Welfare. Cancer data in Australia. Canberra: AlHW; 2019. Report no.: Cat no: CAN 122.

2. Australian Institute of Health and Welfare. Cancer in Australia 2017. Canberra: AlHW; 2017.

3. Australian Institute of Health and Welfare. BreastScreen Australia monitoring report 2014-15. Canberra: AlHW; 2017.

4. Australian Institute of Health and Welfare. Cancer compendium: information and trends by cancer type. 2018.

5. Ng HS, Roder D, Koczwara B, Vitry A. Comorbidity, physical and mental health among cancer patients and survivors: an Australian population-based study. Asia Pac J Clin Oncol. 2017;14(2):e181-92.

6. Cancer Australia. Cancer Australia statement - influencing best practice in breast cancer. Surry Hills: Cancer Australia; 2016.

7. Victorian Department of Health and Human services. Optimal care pathway for women with breast cancer. Viewed January $2002<$ https://www. cancervic.org.au/downloads/health-professionals/optimal-care-pathways/ Optimal_care_pathway_for_women_with_breast_cancer.pdf > .
8. Runowicz CD, Leach CR, Henry NL, Henry KS, Mackey HT, Cowens-Alvarado $\mathrm{RL}$, et al. American Cancer Society/American Society of Clinical Oncology Breast Cancer survivorship care guideline. J Clin Oncol. 2016;34(6):611-35.

9. Grunfeld E, Levine MN, Julian JA, Coyle D, Szechtman B, Mirsky D, et al. Randomized trial of long-term follow-up for early-stage breast cancer: a comparison of family physician versus specialist care. J Clin Oncol. 2006; 24(6):848-55

10. Der Vartanian C, Anderson K, Evans K, Anderiesz C, Giles C, Zorbas H. A new model supporting best practice follow-up care for early breast cancer in Australia: shared follow-up care for early breast cancer. South Australia: Cancer Survivorship 2017; 2017.

11. Hudson SV, Miller SM, Hemler J, Ferrante JM, Lyle J, Oeffinger KC, et al. Adult cancer survivors discuss follow-up in primary care: 'not what I want, but maybe what I need'. Ann Fam Med. 2012;10(5):418-27.

12. Neuman HB, Jacobs EA, Steffens NM, Jacobson N, Tevaarwerk A, Wilke LG, et al. Oncologists' perceived barriers to an expanded role for primary care in breast cancer survivorship care. Cancer Med. 2016;5(9):2198-204.

13. Chan RJ, Downer TR. Cancer nurses can bridge the gap between the specialist cancer care and primary care settings to facilitate shared-care models. Cancer Nurs. 2018;41(2):89-90.

14. Cancer Council Australia. Living well after cancer. A guide for people with cancer, their families and friends. 2018.

15. Cancer Council Australia. Optimal care pathway for women with breast cancer. 2016.

16. Bandura A, National Inst of Mental Health. Prentice-Hall series in social learning theory. Social foundations of thought and action: a social cognitive theory. Englewood Cliffs: Prentice-Hall, Inc; 1986.

17. Bandura A, Adams NE, Beyer J. Cognitive processes mediating behavioral change. J Pers Soc Psychol. 1977:35(3):125-39.

18. DoHA, Flinders University. Capabilities for supporting prevention and chronic condition self-management. Canberra: Department of Health and Ageing and Flinders University; 2009.

19. Taylor K, Chan RJ, Monterosso L. Models of survivorship care provision in adult patients with haematological cancer: an integrative literature review. Support Care Cancer. 2015;23(5):1447-58.

20. Chan RJ, Button E, Thomas A, Gates P, Yates P. Nurses attitudes and practices towards provision of survivorship care for people with a haematological cancer on completion of treatment. Support Care Cancer. 2018;26(5):1401-9.

21. Chan RJ, Yates P, Li Q, Komatsu H, Lopez V, Thandar M, et al. Oncology practitioners' perspectives and practice patterns of post-treatment cancer survivorship care in the Asia-Pacific region: results from the STEP study. BMC Cancer. 2017;17(1):715

22. Molassiotis A, Yates P, Li Q, So WKW, Pongthavornkamol K, Pittayapan $P$, et al. Mapping unmet supportive care needs, quality-of-life perceptions and current symptoms in cancer survivors across the AsiaPacific region: results from the International STEP Study. Ann Oncol. 2017;28(10):2552-8

23. Wallace A, Downs E, Gates P, Thomas A, Yates P, Chan RJ. Provision of survivorship care for patients with haematological malignancy at completion of treatment: a cancer nursing practice survey study. Eur J Oncol Nurs. 2015;19(5):516-22.

24. Bellg AJ, Borrelli B, Resnick B, Hecht J, Minicucci DS, Ory M, et al. Enhancing treatment fidelity in health behavior change studies: best practices and recommendations from the $\mathrm{NIH}$ Behavior Change Consortium. Health Psychol. 2004;23(5):443-51.

25. Robb SL, Burns DS, Docherty SL, Haase JE. Ensuring treatment fidelity in a multi-site behavioral intervention study: implementing NIH Behavior Change Consortium recommendations in the SMART trial. Psycho-oncology. 2011;20(11):1193-201.

26. Brady MJ, Cella DF, Mo F, Bonomi AE, Tulsky DS, Lloyd SR, et al. Reliability and validity of the Functional Assessment of Cancer Therapy-Breast qualityof-life instrument. J Clin Oncol. 1997;15(3):974-86.

27. Chopra I, Kamal KM. A systematic review of quality of life instruments in long-term breast cancer survivors. Health Qual Life Outcomes. 2012;10:14.

28. Hertzog MA. Considerations in determining sample size for pilot studies. Res Nurs Health. 2008;31(2):180-91

29. Julious S. Sample size of 12 per group rule of thumb for pilot study. Pharm Stat. 2005;4:287-91. 
30. Jenkinson C, Coulter A, Bruster S. The Picker Patient Experience Questionnaire: development and validation using data from in-patient surveys in five countries. Int J Qual Health Care. 2002;14(5):353-8.

31. Jenkinson C, Coulter A, Reeves R, Bruster S, Richards N. Properties of the Picker Patient Experience questionnaire in a randomized controlled trial of long versus short form survey instruments. J Public Health Med. 2003;25(3): 197-201.

32. Rutishauser I, Webb K, Abraham B, Allsopp R. Evaluation of short dietary questions from the 1995 National Nutrition Survey. Canberra: Australian Government Department of Health and Ageing; 2001.

33. Australian Institute of Health and Welfare. The active Australia survey: a guide and manual for implementation, analysis and reporting. Canberra: AlHW; 2003.

34. Craig CL, Marshall AL, Sjostrom M, Bauman AE, Booth ML, Ainsworth BE, et al. International physical activity questionnaire: 12-country reliability and validity. Med Sci Sports Exerc. 2003;35(8):1381-95.

35. de Souza JA, Yap BJ, Hlubocky FJ, Wroblewski K, Ratain MJ, Cella D, et al. The development of a financial toxicity patient-reported outcome in cancer: the COST measure. Cancer. 2014;120(20):3245-53.

36. de Souza JA, Yap BJ, Wroblewski K, Blinder V, Araújo FS, Hlubocky FJ, et al. Measuring financial toxicity as a clinically relevant patient-reported outcome: the validation of the COmprehensive Score for financial Toxicity (COST). Cancer. 2017;123(3):476-84.

\section{Publisher's Note}

Springer Nature remains neutral with regard to jurisdictional claims in published maps and institutional affiliations.

Ready to submit your research? Choose BMC and benefit from:

- fast, convenient online submission

- thorough peer review by experienced researchers in your field

- rapid publication on acceptance

- support for research data, including large and complex data types

- gold Open Access which fosters wider collaboration and increased citations

- maximum visibility for your research: over $100 \mathrm{M}$ website views per year

At $\mathrm{BMC}$, research is always in progress.

Learn more biomedcentral.com/submissions 